\title{
Modern Development for the Improvement of Accuracy of Nigerian Coordinate Transformation Process Using the Adapted NTv2 Model: The Critical Issues of the Mathematical Algorithm
}

\author{
Lawrence Hart1, Kurotamuno Peace Jackson1, Francis Ifeanyi Okeke² \\ ${ }^{1}$ Department of Surveying and Geomatics, Rivers State University, Port Harcourt, Nigeria \\ ${ }^{2}$ Department of Geoinformatics and Surveying, University of Nigeria, Enugu, Nigeria \\ Email: Lawrence.hart@ust.edu.ng
}

How to cite this paper: Hart, L., Jackson, K.P. and Okeke, F.I. (2020) Modern Development for the Improvement of Accuracy of Nigerian Coordinate Transformation Process Using the Adapted NTv2 Model: The Critical Issues of the Mathematical Algorithm. International Journal of Geosciences, 11, 768-781.

https://doi.org/10.4236/ijg.2020.1111039

Received: August 24, 2020

Accepted: November 27, 2020

Published: November 30, 2020

Copyright $\odot 2020$ by author(s) and Scientific Research Publishing Inc. This work is licensed under the Creative Commons Attribution International License (CC BY 4.0).

http://creativecommons.org/licenses/by/4.0/

\begin{abstract}
Changing coordinates using appropriate mathematical models from one reference system to another may be influenced if the operation requires the change of datum. A set of transformation parameters has been adopted for Nigeria. However, the critical concern usually associated with the problem of transformation of coordinates is the issue of recoverability of the original values of transformed coordinates. The recursive effect of variables associated with spatial problems can be aptly modelled with an appropriate algorithm that set out a process to achieve a definite output. Consequently, the main thrust of this paper is to highlight the critical elements of the mathematical algorithm associated with the National Transformation Version 2 (NTv2) model adapted for the Nigerian Datum Transformation process. The adapted NTv2 model adopts the bi-linear interpolation approach and the covariance function obtained were used to generate transformation elements in latitude $\left(\Delta \varphi_{p}\right)$ and longitude $\left(\Delta \lambda_{p}\right)$ and corresponding accuracies at the lattice nodes. The mathematical algorithm of this adapted NTv2 model underscores the likely attainment of better and significant values and statistical indicator of the improved accuracy as the average shift values for latitude and longitude for any transformed points in Nigeria. This capability makes the mathematical algorithm to be adaptable and fit for the purpose of the transformation process. The improvement in the positional accuracy is directly attributable to the application of the NTv2 model which provides a flexible and robust system of modelling any inherent systematic error in the national network.
\end{abstract}




\section{Keywords}

Transformation Parameters, Geodetic Datum, NTv2 Model, WGS84

\section{Introduction}

In view of the propagation of systematic errors in measurement and computational operations terrestrial geodetic methods are intricately subject to errors and create distortion which spread into the reference coordinate datum. These apparent distortions in the local geodetic networks, affect mainly the scale and orientation of the network [1] [2]. These errors can be truly modelled by a geometric conformal transformation mathematical algorithm, this can be applied in either micro or macro areas and in most cases as it affects national networks. In view of the need to use a defined unambiguous model in solving the problem of determining transformation parameters for datum transformation, [3] [4] restated that the issue of transformation parameters determination, requires a reliable and theoretically sound approach to the transformation of assembled data with a primary objective to preserve the veracity and topology of prevailing datasets and secondly, to ensure that indistinguishable transformation results are attained nonetheless of who performs the transformation. This indeed brings to the fore the seriousness of a well-developed algorithm to achieve the intended solution. Again, he posits that the transformation problem becomes more intricate if there is a variation in the original data and it is considered beneficial to improve spatial accuracy by eliminating part or all of the distortion (variation) through the transformation process demonstrated in the mathematical algorithm. Whereas, if the geodetic network does not have an inherent distortion, the geometric model of transformation parameters determination enclosed in an appropriate algorithm being adopted would be correspondingly representative across the spatial region and a seamless transformation would result [5] [6]. This notwithstanding, several related works have demonstrated the use of other mathematical models in solving the problem of distortion and systematics errors in coordinate data sets. These alternatives, provide a reliable solution at the same quality level, but where improved computational performance and adequacy of modelling distortion is required, the adapted Ntv2 model becomes a veritable tool. Further to this, when the common points of the two datum surfaces that provide the variables are not evenly distributed, more robust grid-based interpolation techniques such as the Ntv2 model will be appropriate for the determination of datum transformation values with accompanying transformation accuracy [7] [8].

The accuracy and reliability of the associated estimated parameters determined depend not only on the choice of the transformation model, but can be greatly influenced by the location and spread of the common points, especially for the conformal models. For a firm parameter solution, it is important that the 
locations are spatially well distributed. Similarly, [9] agrees that transformation of coordinate that involves non-homogenous systems are greatly enhanced with the deployment of grid interpolation techniques and geometric model, this combination provides better accuracy of the process output. It will have the ease to generate datum shift values for any location within the study area, thereby facilitating data integration of varying datum. [10] asserts that this kind of transformation became important particularly after the development of high precision Global Navigation Satellite System (GNSS), in order to maintain the same relationship over local datum. This will be very important and valuable for the accurate location of boundaries, environmental engineering designs, geomodelling of the earth and near-earth surfaces and optimization of positioning and navigation systems [8] [6] [11].

In our quest to achieve improved accuracy of the datum transformation process of the Nigerian coordinate system, this work will examine the mathematical requirements cased in the algorithmic framework for the deployment of the adapted National Transformation Version 2 Model (NTv2) for Nigeria. Its uniqueness as a grid-based technique demonstrates an improvement in the accuracy of the positional values of the transformed points on the earth or near-earth surfaces within the Nigerian geodetic network as [11] highlights the range of the values of the shift accuracy of transformed coordinates as obtained from this adapted model with a maximum value of $0.184 \mathrm{~m}$ in latitude and 0.281 $\mathrm{m}$ in longitude respectively. This model was adapted for the Nigerian Datum Transformation Process for improved accuracy considering the suspect of inherent distortion in the definition of the Nigerian geodetic origin.

\section{Conceptual Review of Interpolation Techniques}

The process of interpolation encompasses making approximations of the interpolated value of an attribute variable at points where there is no existing information by using data for a sample of the point where we do have information. Several interpolation techniques abound and applicable in other areas of science and engineering and they are fundamentally based on [12] and [13], three main categories: deterministic, probabilistic and other methods. The numerical method of interpolation using Laplace's equation [3], interpolation by the use of geometric distance by weighting functions [14]. [15] has also used irregularly gridded sea level data in the coastal zone to model/interpolate sea surface topography using the least-squares collocation technique. [16] described the technique as a process of determining a function by fitting an approximation of an analytical function to a given number of linear functions. It is predominantly deployed in geospatial sciences in estimating variables at points in which quantities are not available, using the information of known points (signals). In the same vein, the National Transformation Version 2 model (NTv2) is a grid-based interpolation technique [4]. The NTv2 method adopts binary files to accomplish accurate datum shifts from one geodetic reference system to another. An NTv2 
file comprises the changes between the two reference systems as geographic seconds in a grid. The process of bi-linear interpolation is used to compute the exact coordinates for a point in the target reference ellipsoid system and generate transformation accuracy parameters for the computed points.

\section{Materials and Methods}

\subsection{Materials Used}

The Office of the Surveyor-General of the Federation (OSGoF) [17] has provided coordinates of 50 geodetic stations of common points in Nigeria which serve as an existing dataset as shown in Table 1 [11]. This is in addition to the derived values of the ellipsoidal height in Minna datum as self-generated data as highlighted in Table 2 was used for this study.

Table 1. Specimen of Cartesian coordinates of Minna datum and WGS 84 datum (Source: Office of the surveyor general of the federation of Nigeria).

\begin{tabular}{|c|c|c|c|c|c|c|c|}
\hline$S / N$ & $\begin{array}{l}\text { STATION } \\
\text { NAME/ID }\end{array}$ & $\begin{array}{c}\text { MINNA } \\
\text { LATITUDE } \\
\text { DECIMAL } \\
\text { DEGREES }\end{array}$ & $\begin{array}{c}\text { MINNA } \\
\text { LONGITUDE } \\
\text { DECIMAL } \\
\text { DEGREE }\end{array}$ & $\begin{array}{c}\text { MINNA } \\
\text { ELLIPSOIDAL } \\
\text { HEIGHT }\end{array}$ & $\begin{array}{c}\text { WGS84 } \\
\text { LATITUDE } \\
\text { DECIMAL } \\
\text { DEGREES }\end{array}$ & $\begin{array}{c}\text { WGS84 } \\
\text { LONGITUDE } \\
\text { DECIMAL } \\
\text { DEGREE }\end{array}$ & $\begin{array}{c}\text { WGS } 84 \\
\text { ELLIPSOIDAL } \\
\text { HEIGHT (M) }\end{array}$ \\
\hline 1 & A 10 & 9.319181 & 12.23059028 & 274.116 & 9.31946547 & 12.2299544 & 287.036 \\
\hline 2 & A 24 & 10.60364 & 11.34032833 & 610.163 & 10.6037971 & 11.3396676 & 624.625 \\
\hline 3 & A16 & 10.12471 & 12.37651389 & 773.545 & 10.1249196 & 12.375878 & 786.604 \\
\hline 4 & A39 & 11.28878 & 10.41809139 & 479.759 & 11.2888627 & 10.4173975 & 495.499 \\
\hline 5 & $\mathrm{C} 21$ & 6.968268 & 9.247406944 & 329.453 & 6.96865594 & 9.24683417 & 344.608 \\
\hline 6 & $\mathrm{C} 16$ & 6.136606 & 9.027243056 & 613.763 & 6.13706426 & 9.02665414 & 628.430 \\
\hline 7 & C32 & 7.757921 & 10.12114583 & 377.183 & 7.75822143 & 10.1205124 & 391.825 \\
\hline 8 & CFL56 & 11.85322 & 13.1170425 & 344.927 & 11.853237 & 13.1164306 & 357.405 \\
\hline 9 & CFH66 & 6.172683 & 6.750818611 & 39.549 & 6.17314447 & 6.7501325 & 57.368 \\
\hline 10 & CFA 33A & 6.626514 & 3.323891389 & 47.165 & 6.62690544 & 3.32312688 & 70.412 \\
\hline 45 & L040 & 9.635833 & 6.516389722 & 281.108 & 9.63602803 & 6.51564808 & 301.648 \\
\hline 46 & N032 & 9.105738 & 7.202294444 & 689.447 & 9.10598537 & 7.20157031 & 708.804 \\
\hline 47 & A001 & 9.188534 & 12.49675972 & 205.402 & 9.18882886 & 12.4961279 & 217.938 \\
\hline 48 & U72 & 7.453301 & 5.871579444 & 638.312 & 7.45366794 & 5.87108417 & 658.509 \\
\hline 49 & C036 & 7.998495 & 10.99365694 & 529.928 & 7.99877698 & 10.9930426 & 543.625 \\
\hline 50 & A 21 & 10.45509 & 11.62860417 & 744.895 & 10.455269 & 11.6279517 & 758.963 \\
\hline 51 & $\mathrm{H} 2$ & 7.33078 & 9.053321667 & 494.999 & 7.33115025 & 9.05274885 & 510.712 \\
\hline 52 & L41 & 9.585434 & 6.507209444 & 235.874 & 9.58563389 & 6.50646808 & 256.406 \\
\hline 53 & N107 & 9.492585 & 6.775244167 & 544.930 & 9.49279582 & 6.77451014 & 565.051 \\
\hline
\end{tabular}


Table 2. Specimen of computed undulation correction and Minna datum ellipsoidal height.

\begin{tabular}{|c|c|c|c|c|c|c|}
\hline $\mathrm{S} / \mathrm{N}$ & $\begin{array}{l}\text { STATION } \\
\text { NAME/ID }\end{array}$ & $\begin{array}{c}\text { MINNA } \\
\text { ORTHOMETRIC } \\
\text { HEIGHT }\end{array}$ & $\begin{array}{c}\text { WGS } 84 \\
\text { ELLIPSOIDAL } \\
\text { HEIGHT (M) }\end{array}$ & $\begin{array}{l}\text { PRIME VERTICAL } \\
\text { (MINNA DATUM) }\end{array}$ & $\Delta \mathrm{H}$ & $\begin{array}{c}\text { MINNA } \\
\text { ELLIPSOIDAL } \\
\text { HEIGHT } H_{\text {MINNA }}\end{array}$ \\
\hline 1 & A10 & 268.127 & 287.0361 & $6,378,818.17851$ & 12.92059779 & 274.1155022 \\
\hline 2 & A 24 & 605.629 & 624.6253 & $6,378,983.96092$ & 14.46199188 & 610.1633081 \\
\hline 3 & A16 & 768.768 & 786.6037 & $6,378,919.74827$ & 13.05853023 & 773.5451698 \\
\hline 4 & A39 & 474.746 & 495.4991 & $6,379,080.73508$ & 15.73962794 & 479.7594721 \\
\hline 5 & $\mathrm{C} 21$ & 324.6 & 344.608 & $6,378,568.51743$ & 15.15490663 & 329.4530934 \\
\hline 6 & $\mathrm{C} 16$ & 607.473 & 628.4296 & $6,378,497.10286$ & 14.66658108 & 613.7630189 \\
\hline 7 & $\mathrm{C} 32$ & 373.029 & 391.8245 & $6,378,644.54154$ & 14.64100334 & 377.1834967 \\
\hline 8 & CFL56 & 340.68 & 357.4045 & $6,379,164.77329$ & 12.47759485 & 344.9269052 \\
\hline 9 & CFH66 & 37.214 & 57.368 & $6,378,500.01578$ & 17.81909126 & 39.54890874 \\
\hline 10 & CFA 33A & 47.093 & 70.412 & $6,378,538.09317$ & 23.2469371 & 47.1650629 \\
\hline 11 & D29 & 505.549 & 526.7924 & $6,379,095.21757$ & 22.77818301 & 504.014217 \\
\hline 12 & D17 & 325.526 & 350.1121 & $6,379,005.62972$ & 23.64860407 & 326.4634959 \\
\hline 13 & $\mathrm{H} 4$ & 341.778 & 362.903 & $6,378,615.15240$ & 16.4177838 & 346.4852162 \\
\hline 14 & H5 & 279.859 & 300.431 & $6,378,619.98762$ & 15.96341579 & 284.4675842 \\
\hline 15 & $\mathrm{H} 11$ & 239.394 & 259.5107 & $6,378,695.66251$ & 16.70430421 & 242.8063958 \\
\hline 16 & L10 & 172.987 & 197.892 & $6,378,590.38326$ & 23.7087912 & 174.1832088 \\
\hline 17 & L18 & 404.216 & 430.3807 & $6,378,727.29593$ & 22.8215704 & 407.5591296 \\
\hline 18 & L16 & 498.209 & 523.7774 & $6,378,659.46205$ & 22.65178951 & 501.1256105 \\
\hline 19 & MW606 & 90.472 & 105.326 & $6,378,422.05140$ & 14.4719753 & 90.8540247 \\
\hline 20 & N25 & 570.12 & 591.959 & $6,378,780.00431$ & 18.09340511 & 573.8655949 \\
\hline 21 & N102 & 449.616 & 471.778 & $6,378,857.51769$ & 20.48091504 & 451.297085 \\
\hline 22 & N127 & 754.644 & 776.0028 & $6,379,210.28307$ & 20.5385254 & 755.4642746 \\
\hline 49 & C036 & 524.471 & 543.625 & $6,378,669.28662$ & 13.69658631 & 529.9284137 \\
\hline 50 & A 21 & 740.331 & 758.963 & $6,378,963.74417$ & 14.06832624 & 744.8946738 \\
\hline 51 & $\mathrm{H} 2$ & 489.83 & 510.712 & $6,378,602.43$ & 15.71285083 & 494.9991492 \\
\hline 52 & L41 & 234.356 & 256.406 & $6,378,850.857$ & 20.53221747 & 235.8737825 \\
\hline 53 & N107 & 543.706 & 565.051 & $6,378,839.361$ & 20.12052499 & 544.930475 \\
\hline
\end{tabular}

\subsection{Computation of the Ellipsoidal Height Difference}

The Minna datum (Clarke 1880) ellipsoidal height $\left(h_{\text {CLK1880 }}\right)$ of a station is computed directly from the corresponding WGS84 ellipsoidal height $\left(h_{\text {WGs84 }}\right)$ in Equation (1). The 5-parameter generalized form of Molodensky Standard formula [18] as shown in Equation (2) was used to compute the approximate change difference in ellipsoidal height $\left(\Delta h_{\mathrm{WGS} 84}\right)$ which is applied to the WGS84 ellipsoidal height $\left(h_{\mathrm{WGS} 84}\right)$ to convert it to the corresponding Minna datum value $\left(h_{\text {CLK1880 }}\right)$ [19] [20]. The values are shown in Table 2. 


$$
h_{\mathrm{CLK} 1880}=h_{\mathrm{WGS} 84}+\Delta h_{\mathrm{WGS} 84}
$$

where,

$$
\begin{aligned}
\Delta h_{\mathrm{WGS} 84}= & \Delta \chi \cos \varphi \cos \lambda+\Delta Y \cos \varphi \sin \lambda+\Delta z \sin \varphi \\
& -\left(\Delta a \frac{a}{V}\right)+\left(\Delta f \frac{b}{a}\right) V \sin ^{2} \varphi
\end{aligned}
$$

$\varphi, \lambda=$ WGS84 coordinates of the station

$\Delta x, \Delta y, \Delta z=$ Datum shifts to transform WGS84 datum to Minna datum

$\Delta a, \Delta f=$ (Minna minus WGS84) semi-major and flattening respectively

$a_{\mathrm{WGS} 84}=$ Semi-Major Axis Radius of WGS84 Ellipsoid $=6,378,137 \mathrm{~m}$

$f_{\mathrm{WGS} 84}=$ Flattening of WGS84 ellipsoid $=1 / 298.257223563$

$a_{\text {MINNA }}=6,378,249.145 \mathrm{~m}$

$f_{\mathrm{MINNA}}=1 / 293.465$

$\Delta a=112.145 \mathrm{~m}$

$\Delta f \times 10^{4}=0.54750714(\Delta f=0.000054750714)$

$\Delta x=93.708 \mathrm{~m}$

$\Delta y=92.626 \mathrm{~m}$

$\Delta z=-121.33 \mathrm{~m}$

$$
V=\text { Radius of curvature of the Prime Vertical }=\frac{a}{\left(1-e^{2} \sin ^{2} \varphi\right)^{1 / 2}}
$$

$$
\begin{gathered}
b / a=1-f \\
e^{2}=2 f-f^{2}
\end{gathered}
$$

\subsection{Mathematical Model and Algorithm for the Development of Nigerian Adapted NTv2 Model Grid Shift File}

The National Transformation Version 2 (NTv2) is a mathematical format that is built on the components of grid shift file that houses the coordinate shift values as determined at the grid nodes of a regular lattice. This format was developed by the Geodetic Survey Division of Geomatics Canada [4]; in this research, it was adapted to implement the transformation of coordinates between Minna datum (Clarke 1880) and WGS84 in Nigeria [6]. The nodes in a grid shift file are arranged in rows initialized from the south east (lower right) corner, and finishing in the North West (upper left) corner. The shift values ( $\Delta \varphi$ and $\Delta \lambda$ derived from Equation (6)) of the irregularly spaced geodetic network are modelled onto a regular grid surface. The mathematical algorithm provided the framework for all the complexities and processing in the creation of these grid files, and the set of instruction accompanying the model adapted for the transformation process ensures the interpolation of the required shifts and perform a simple addition to complete the transformation operation [11].

The bi-linear interpolation was used in this research to determine transformation component in latitude $\left(\Delta \varphi_{p}\right)$ and longitude $\left(\Delta \lambda_{p}\right)$. The algorithm is given as [11] [16]: 


$$
\delta \varphi_{P}=a_{0}+a_{1} V+a_{2} W+a_{3} V W
$$

where

$$
\begin{gathered}
a_{0}=\delta \varphi_{1} \\
a_{1}=\delta \varphi_{2}-\delta \varphi_{1} \\
a_{2}=\delta \varphi_{4}-\delta \varphi_{1} \\
a_{3}=\delta \varphi_{1}+\delta \varphi_{3}-\delta \varphi_{2}-\delta \varphi_{4} \\
V=\left(\frac{\lambda_{P}-\lambda_{1}}{\lambda_{2}-\lambda_{1}}\right) \\
W=\left(\varphi_{P}-\varphi_{1}\right) /\left(\varphi_{3}-\varphi_{1}\right)
\end{gathered}
$$

where

$a_{0}, a_{1}, a_{2}, a_{3}$ are interpolation parameters.

$V, W$ is interpolation scale factors.

From the foregoing, the relevant distortion components in latitude and longitude are represented as $\left(\delta \varphi_{1}, \delta \varphi_{2}, \delta \varphi_{3}, \delta \varphi_{4} ; \delta \lambda_{1}, \delta \lambda_{2}, \delta \lambda_{3}, \delta \lambda_{4}\right)$. The latitude and longitude of the interpolated point is represented as $\left(\varphi_{P}\right.$ and $\left.\lambda_{P}\right)$. Similarly, the value of the longitude transformation component of the interpolated point can be determined using Equations (7)-(12). However, the $\delta \varphi$ terms in the equations was substituted with the equivalent $\delta \lambda$ terms from the grid. The rudimentary contents of the Nigerian grid shift file depict the upper and lower limit of the latitude and longitude frame. The interval for the grid in seconds of arc for latitude and longitude. Equations (13) and (14) demonstrate the determination of the total numbers of rows and column within the limit of the study area. The grid shift values in relation to the determined rows and columns are also generated in seconds (rows, columns) [6] [5].

No. of Rows

$$
\begin{gathered}
1+\left(\left(\varphi_{\text {Upper }}-\varphi_{\text {Lower }}\right) / \Delta \varphi\right) \\
M=1+\operatorname{integer}(55800-10800) / 30
\end{gathered}
$$

No. of Columns

$$
\begin{gathered}
1+\left(\left(\lambda_{\text {Upper }}-\lambda_{\text {Lower }}\right) / \Delta \lambda\right) \\
N(\text { No of Columns })=1+(55800-7200) / 30
\end{gathered}
$$

The process of generating the shift (distortions) values from the grid nodes for the purpose of interpolation is given is as shown Equations (15) and (16):

$$
\begin{gathered}
\text { row } i=1+\operatorname{Integer} \frac{\varphi_{p}-\varphi_{\text {Lower }}}{\Delta \varphi} \\
\text { Column } j=1+\text { Integer } \frac{\lambda_{p}-\lambda_{\text {Lower }}}{\Delta \lambda}
\end{gathered}
$$

The $i$ th and $j$ th values for the rows and columns are generated thus:

$$
i=1+(33549.051-10800) / 30=1+22749.051 / 30
$$




$$
\begin{gathered}
i=759 \\
j=1+(44030.250-7200) / 30=1+1228 \\
j=1229
\end{gathered}
$$

where

$\varphi_{p}, \lambda_{p}$ the latitude and longitude of the interpolated point

$\varphi_{\text {Lower }}, \lambda_{\text {Lower }}$ lower latitude and longitude extent of the grid

$\Delta \varphi, \Delta \lambda$ Latitude and longitude interval between grid nodes

This process is carried out for the remaining grid nodes encompassing the point of interest within the grid file. Again, the computation of coordinate of grid nodes are determined using Equations (17) and (18) in terms of latitude and longitude. The transformed coordinates of the points of interest was derived as shown in Equations (19) and (20).

$$
\begin{gathered}
\varphi_{1}=\varphi_{\text {Lower }}+(i-1) * \Delta \varphi \\
\lambda_{1}=\lambda_{\text {Lower }}+(j-1) * \Delta \lambda \\
\varphi_{\text {WGS84 }}=\varphi_{p}+\delta \varphi_{p}
\end{gathered}
$$

Also,

$$
\lambda_{\mathrm{WGS} 84}=\lambda_{p}+\delta \lambda_{p}
$$

Figure 1 diagrammatically demonstrate the interpolation of transformation components from a typical grid file as expressed in the mathematical algorithm for adapted NTv2 model for Nigeria.

In another vein, covariance function and transformation accuracy at grid nodes was derived using the algorithm of the haversine model. The associated distances between data points from the grid node in addition with the surrounding data points is given in Equation (21):

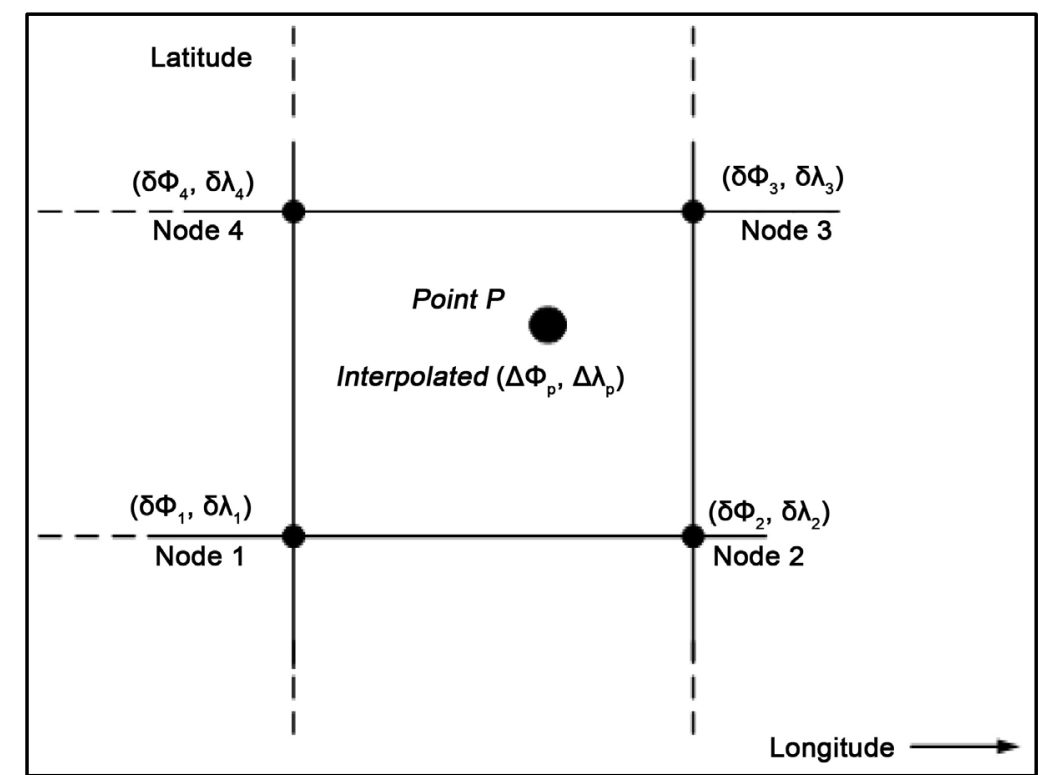

Figure 1. Interpolating transformation components from a grid file. 


$$
d=2 r \sin ^{-1}\left(\sqrt{\sin ^{2}}\left(\frac{\varphi_{2}-\varphi_{1}}{2}+\cos \left(\varphi_{1}\right) \cos \left(\varphi_{2}\right) \sin ^{2}\left(\frac{\lambda_{2}-\lambda_{1}}{2}\right)\right)\right)
$$

where, $d$ is the distance between the two points along the great circle of the sphere, $r$ is the radius of the sphere (sometimes referred to as the mean radius of the earth), $\varphi_{1}, \varphi_{2}$ Latitude of locations 1 and $2, \lambda_{1}, \lambda_{2}$ Longitude of locations 1 and 2.

Consequently, the transformation accuracy computation at each grid node is based on the model proposed by [21] [22] as shown in Equation (22). It gives us the reliability of the transformation process.

$$
\sigma=\sqrt{\frac{\Sigma w_{i}^{2} \Sigma\left(\delta_{i}-\bar{\delta}\right)^{2}}{\left(\Sigma w_{i}\right)^{2}(n-1)}}
$$

where, $\sigma$ is transformation accuracy, $\bar{\delta}$ is the computed shift component at the grid node (by collocation), $\delta_{i}$ is the known shift component at each data point (i), $w_{1}$ is the weight derived from the covariance function and based on the distance of point from the interpolation point, $n$ is the number of data points.

\section{Results and Discussions}

The specimen values of the prime vertical, the undulation correction and computed Minna ellipsoidal heights based on Minna Datum are shown in Table 2. The values of the Minna ellipsoidal heights create the homogeneity of the datasets of the two-reference surface. This is required for datum transformation process. In view of the grid-based capability of the adapted model, the computation of essential grid parameters includes the determination of the total number of rows and columns that encloses the upper and lower limit of the study area (Nigeria) was generated. We have 1501 rows (lines of latitude) and 1621 columns (lines of longitude). The grid nodal values was derived using the ith and $j$ th of the shift distortion model as in Equations (15) and (16); Node $1=N \times(i-$ $1)+j=1621 \times(759-1)+1229 ;$ Node $1=1229947 ;$ Node $2=$ Node $1+1 ;$ Node $2=1229947+1=1229948$; Node $3=$ Node $1+\mathrm{N} ;=1229947+1621 ;$ Node $3=$ 1231568; Node $4=$ Node $3+1$; Node $4=1231568+1=1231569$. The coordinate of the initial grid node point (in seconds) is given as $\varphi_{1}=33540 " ; \lambda_{1}=44040 "$. The values of the interpolation scale factor $V=-0.3291663333$ and $W=0.3017$ were obtained. Hence, the latitude and longitude interpolation parameters required for the seamless transformation process are:

$$
\begin{aligned}
a_{0}=\delta \varphi_{1}=-0.250228 \\
a_{1}=\delta \varphi_{2}-\delta \varphi_{1}=-0.208187-0.250228=0.042041 \\
a_{2}=\delta \varphi_{3}-\delta \varphi_{1}=-0.249185-0.250228=0.001043 \\
a_{3}=\delta \varphi_{1}+\delta \varphi_{4}-\delta \varphi_{2}-\delta \varphi_{3} \\
=-0.250228-0.173237-0.042041-0.001043 \\
=-0.466549
\end{aligned}
$$

$$
a_{0}=\delta \lambda_{1}=0.075285
$$




$$
\begin{aligned}
a_{1} & =\delta \lambda_{2}-\delta \lambda_{1}=0.099675-0.075285=0.02439 \\
a_{2} & =\delta \lambda_{3}-\delta \lambda_{1}=0.079317-0.075285=0.004032 \\
a_{3} & =\delta \lambda_{1}+\delta \lambda_{4}-\delta \lambda_{2}-\delta \lambda_{3} \\
& =0.075285+0.156117-0.099675-0.079317 \\
& =0.05241
\end{aligned}
$$

Equation (6) was used to compute the interpolated shift (distortion) value (latitude);

$$
\begin{aligned}
\delta \varphi_{P}= & -0.250228+0.042041 \times(-0.3291663333)+0.001043 \times 0.3017 \\
& +(-0.466599) \times(-0.3291663333) \times 0.3017 \\
= & -0.250228+(-0.0138384818)+0.0003146731+0.0463377053 \\
= & -0.2174141034
\end{aligned}
$$

This value $\delta \varphi_{P}=-0.2174141034$ is then added to the latitude value of the point of interest in Minna Clarke 1880 reference ellipsoid to obtain the corresponding value of latitude in WGS84 ellipsoid. Similarly, the value of $\delta \lambda_{P}$ is also determined by substituting the values of longitude interpolation parameters substituted in Equation (6). The average shift values for latitude and longitude of Nigeria are -0.252138 " and $0.007462 "$ respectively.

The summary of Nigerian grid shift file depicts the limits and the associated accuracy values that ensures that any point of interest can be determined in terms of the transformation of coordinates [21].

$\begin{array}{ll}\varphi_{\text {Lower }} & 10800.0000 \text { (in seconds) } \\ \varphi_{\text {Upper }} & 55800.0000 \text { (in seconds) } \\ \lambda_{\text {Lower }} & 7200.0000 \text { (in seconds) } \\ \lambda_{\text {Upper }} & 55800.0000 \text { (in seconds) } \\ \Delta \varphi & 30 \text { (in seconds) } \\ \Delta \lambda & 30 \text { (in seconds) } \\ \text { No. of Rows } \quad 1501 \\ \text { No. of Columns } 1621\end{array}$

Number of grid shift values (rows $\times$ columns)

\begin{tabular}{cccc}
\hline$\delta \varphi$ & $\delta \lambda$ & $\sigma \varphi$ & $\sigma \lambda$ \\
\hline-0.250227555 & 0.075285446 & 0.406831 & 0.406524 \\
-0.208187407 & 0.099674602 & 0.406831 & 0.406524 \\
-0.249185285 & 0.079317281 & 0.406831 & 0.406524 \\
\hline
\end{tabular}

The transformed values in a grid shift file relate to a defined transformation path which is denoted as the forward transformation. For the Nigerian grid shift files, the forward transformation path is from Minna (Clarke1880) ellipsoid to WGS84 ellipsoid. The process is thus, create summary of grid shift file; get coordinates of interpolation point; determine sub grid that the point lies within; retrieve shift values of the nearest 4 nodes from grid shift file; use bi-linear interpolation to compute coordinate shift valid, this can be categorized as a minor grid. Add the computed shift to transformed coordinates and transform another 
point, by this, one cycle of the forward transformation process is completed. The transformation of coordinates in the opposite direction (WGS84 to Minna-Clarke 1880) in Nigeria is referred to as a back transformation [5] [21]. In a (back) reverse transformation, coordinates of the interpolation point are in a different datum (WGS84) to the grid nodes (Minna-Clarke1880), so the required shifts cannot be directly interpolated off the grid. Because of this, the back-transformation problem requires an iterative solution. The reverse transformation involves computing the shift components at the approximate Minna-Clarke1880 coordinates of a WGS84 point. Subtracting the shifts computed from the WGS84 coordinates produces a better approximation of the Minna-Clarke1880 coordinates. By updating the initial Minna-Clarke1880 coordinates with the new values and repeating the process three times, the exact Minna-Clarke1880 coordinates of a WGS84 point are produced. The back-transformation process is thus:

1. Initialise Minna-Clarke1880 coordinates:

$$
\begin{aligned}
& \varphi_{\text {Minna-Clarke1880 }}^{\prime}=\varphi_{\text {WGS84 }} \\
& \lambda_{\text {Minna-Clarke1880 }}^{\prime}=\lambda_{\text {WGS84 }}
\end{aligned}
$$

2. Repeat four times:

3. Interpolate shifts ( $\delta \varphi$ and $\delta \lambda)$ at Minna-Clarke1880 point.

4. Compute new Minna-Clarke1880 coordinates:

$$
\begin{aligned}
& \varphi_{\text {Minna-Clarke1880 }}^{\prime}=\varphi_{\mathrm{WGS} 84}-\delta \varphi \\
& \lambda_{\text {Minna-Clarke1880 }}^{\prime}=\lambda_{\text {WGS84 }}-\delta \lambda
\end{aligned}
$$

The outcome of an efficient mathematical algorithm is the development of behind the scene code for the solving of the particular problem. Computer programming language was employed in adding functionality, in a code-behind-file model which has PC version [6]. Figure 2 and Figure 3, shows the screen shot of the user interface for the datum transformation process between the WGS 84 and Minna Datum (based on Clarke 1880 ellipsoid) and the interface for the selection of the grid files for the optimization of the transformation process. These parameters also can be deployed in the development of GIS software to optimize the positioning of features and efficient mapping system.

\section{Conclusion}

The uniqueness of this adapted model is predicated on-grid shift files with corresponding grid shift parameters. [6] [11] asserts that this adapted NTv2 model for Nigeria will provide geospatial users with transformation parameters and accompanying positional accuracy on a regularly spaced grid. The output is conveniently generated using the known components of the four surrounding grid nodes. Notwithstanding the mathematical complexity associated with this adapted model, the results from this approach can be showcased to end-users as a simple file of shifts in terms of latitude \& longitude, emplaced on a regular grid format, which can be used to interpolate the shifts for any other location. Hence, 


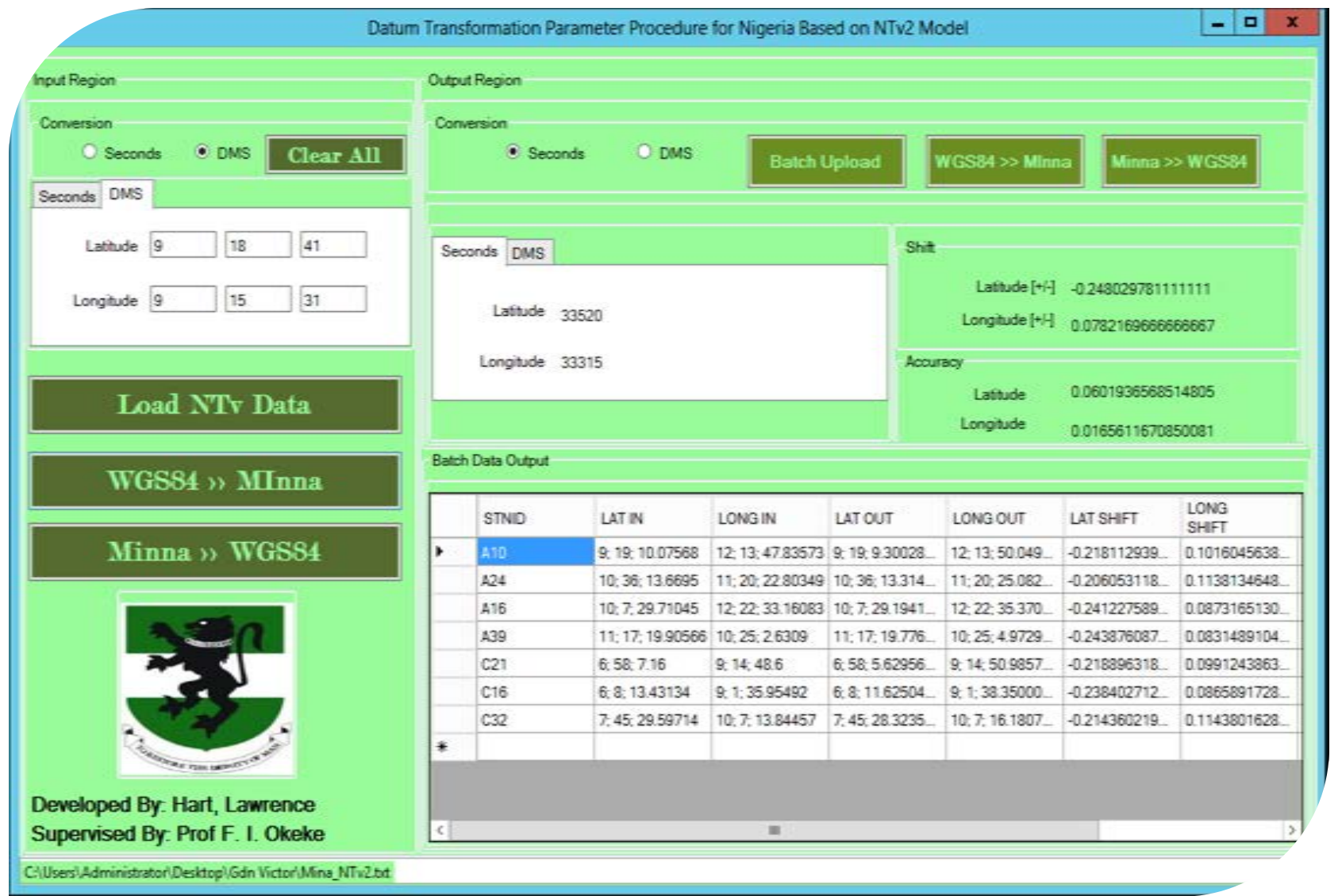

Figure 2. Sample Screen shot PC version (Source: Hart, 2015).

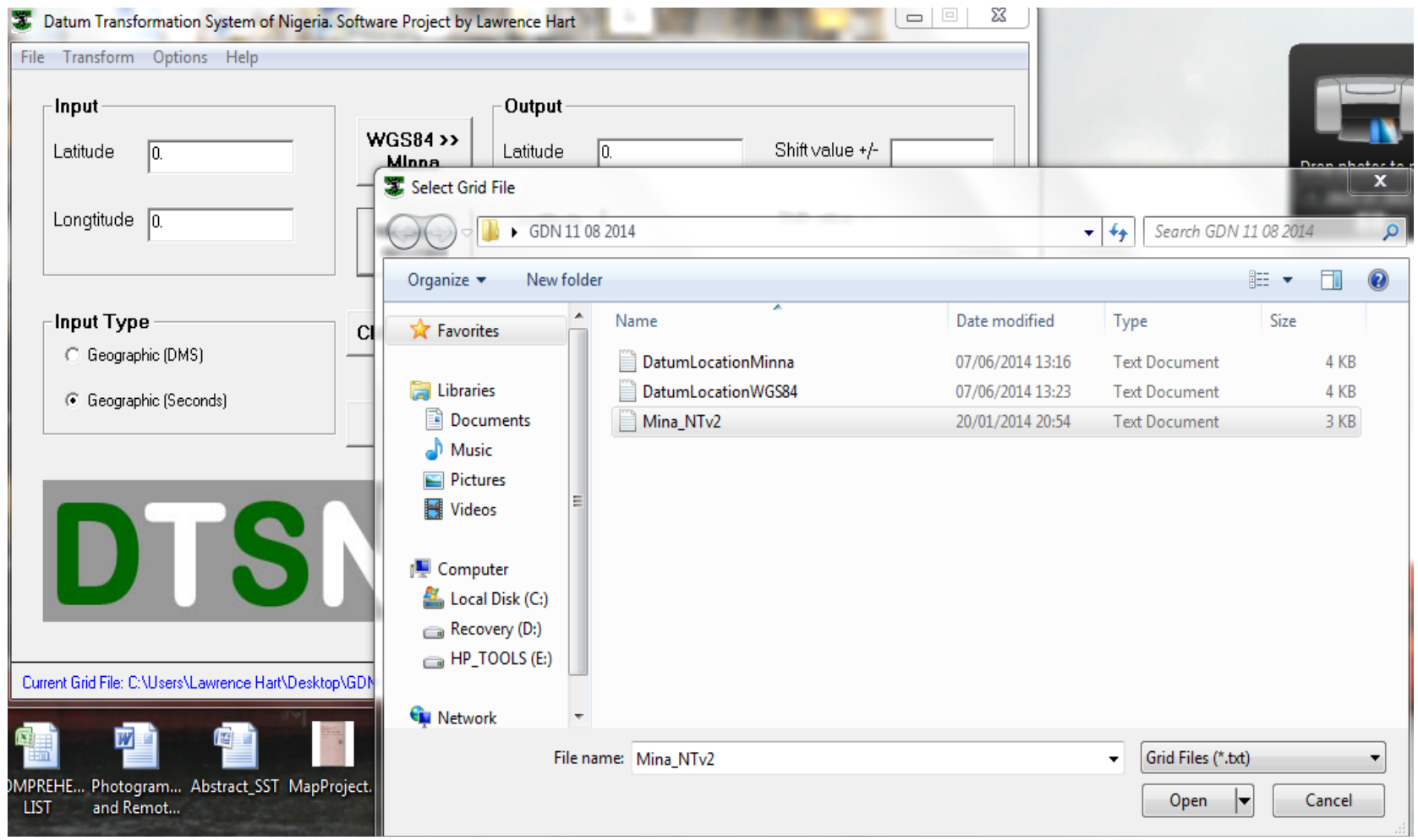

Figure 3. Interface for selecting grid file (Source Hart, 2015). 
the algorithmic propelled in the mathematical model provided the framework for the development of an efficient transformation procedure in line with the objective of this work. These parameters form the framework of the surface modelling of the two-reference datum in other to obtain the corresponding values (coordinate) in its transformed state. Furthermore, the interplay of these grid file data generates the accompanying basis of accuracy determination on point by point basis with no geometric bias. The associated improvement in the positional accuracy as against the use of geometric models of transformation is directly attributable to the application of the adapted NTv2 mathematical algorithm (model) for the Nigerian datum transformation process.

\section{Conflicts of Interest}

The authors declare no conflicts of interest regarding the publication of this paper.

\section{References}

[1] Featherstone, W.E. (1997) A Comparison of Existing Co-Ordinate Transformation Models and Parameters in Australia. Cartography, 26, 13-26.

https://doi.org/10.1080/00050343.1997.10558681

[2] Featherstone, W.E. and Vanicek, P. (1998) The Role of Coordinate Systems, Coordinate and Heights in Horizontal Datum Transformation. The Australia Surveyor, 44, 143-150. https://doi.org/10.1080/00050352.1999.10558789

[3] Dewhurst, W.T. (1990) NADCON, the Application of Minimum Curvature-Derived Surfaces in the Transformation of Positional Data from the North American Datum of 1927 to the North American Datum of 1983. NOAA Technical Memorandum NOS NGS-50, National Ocean Service, U.S. Department of Commerce, Rockville.

[4] Collier, P. (2002) Development of Australia's National GDA94 Transformation Grids. Consultant's Report to the Intergovernmental Committee on Surveying and Mapping Department of Geomatics, Melbourne.

[5] Okeke, F.I. (2014) Coordinate Transformation Parameters and Procedure. Technical Meeting on Adoption of Sustainable Transformation Parameters for Nigeria ( WGS84-Minna Datums), Abuja, 15-17 July 2014, 1-14.

[6] Hart, L. (2015) Development of Datum Transformation Procedure for Nigeria Based on National Transformation Version 2 (NTv2) Model. An Unpublished PhD Thesis, The Department of Geoinformatics and Surveying, University of Nigeria, Enugu Campus, Nigeria..

[7] Uzodinma, V.N. (2005) Improved Minna Datum Geoidal Heights and Origin Definition from a Combination of Astrogeodetic, Geopotential and Satellite Doppler Data. 2nd International Conference of Nigerian Union of Radio \& Planetary Science (NUPRS), Port Harcourt, 23-26 February, 25-40.

[8] Moka, E.C., Agajelu, S.I. and Okeke, F.I. (2006) An Assessment of the Distortions in a Part (K-Chain) of the Nigerian Horizontal Geodetic Network. General Assembly Conference of the Nigeria Association of Geodesy, Lagos, 23-25 August 2006, 15-29.

[9] Iliffe, J. and Lott, R. (2012) Datums and Map Projections for Remote Sensing, GIS and Surveying. 2nd Edition, Whittles Publishing, Dunbeath.

[10] Nwilo, P.C., Fajemirokun, F.A., Ezeigbo, C.U., Oyewusi, A.M. and Ayodele, E.G. 
(2006) Comparison of Some Transformation Procedures. General Assembly Conference of the Nigeria Association of Geodesy, Lagos, 23-25 August 2006, 33-46.

[11] Hart, L. and Okeke, F.I. (2016) Assessment of the Capabilities of the National Transformation Version 2 (NTv2) Model for the Nigerian Datum Transformation. Tropical Environment, 14, 1-14.

[12] Dobesch, H.P., Dumolard, P. and Dryas, I. (2007) Spatial Interpolation for Climate Data: The Use of GIS in Climatology and Meterology Geographical Information Systems Series. ISTE Ltd., London. https://doi.org/10.1002/9780470612262

[13] Tveito, O.E., Wegehenkel, M., Van der Wel, F. and Dobesch, H. (2006) The Use of Geographic Information Systems in Climatology and Metreology-Final COST Action 719 .

[14] Jackson, K.P. (2011) Improved Transformation of the Nigerian Distorted Geodetic Datum into AFREF by Raster Corrections. An Unpublished M.Sc. Thesis, Department of Geoinformatics and Surveying, University of Nigeria, Enugu Campus.

[15] Iliffe, J., Ziebart, M., Cross, P.A., Forsberg, R., Strykowski, G. and Tscherning, C.C. (2003) OSGM02: A New Model for Converting GPS-Derived Heights to Local Height Datums in Great Britain and Ireland. Survey Review, 37, 276-293. https://doi.org/10.1179/sre.2003.37.290.276

[16] Moritz, H. (1980) Advanced Physical Geodesy, Karisruhe Wichmann.

[17] Office of the Surveyor General of the Federation of Nigeria, 2015.

[18] United States Defence Mapping Agency (1987) Supplement to Department of Defence World Geodetic System 1984 Technical Report. Defence Mapping Agency Technical Report No. 8350.2-A, Washington DC.

[19] Fubara, D.M.J. (1995) Improved Determination of Nigerian Geodetic Datum Transformation Parameters for Effective Use of GPS. Quality Control Report for Shell Petroleum Development Company of Nigeria Limited, Port Harcourt.

[20] Marzooqi, Y.Al., Fashir, H. and Syed, I.A. (2005) Derivation of Datum Parameters for Dubai Emirates. FIG Working Week 2005 and GSDI-8, Cairo, 16-21 April 2005, $2-10$.

[21] Junkins, D. and Erickson, C. (1996) Version 2 of the National Transformation between NAD 27 and NAD 83 and Its Importance for GPS Positioning in Canada. Draft Report, Geodetic Survey Division, Geomatics Canada, Ottawa, 18 p. https://doi.org/10.4095/327554

[22] Hampel, F.R., Ronchetti, E.M., Rousseeuw, P.J. and Stahel, W.A. (1986) Robust Statistics: The Approach Based on Influence Functions. John Wiley and Sons, New York. 